\title{
Tracking Age Differences in Neural Distinctiveness across Representational Levels
}

\author{
${ }^{\circledR}$ Malte Kobelt, ${ }^{1,2 *}{ }^{-}$Verena R. Sommer, ${ }^{1 *}{ }^{\circledR}$ Attila Keresztes, ${ }^{3,4}{ }^{\circledR}$ Markus Werkle-Bergner, ${ }^{1 \dagger}$ and \\ ${ }^{\circ}$ Myriam C. Sander ${ }^{1 \dagger}$ \\ ${ }^{1}$ Center for Lifespan Psychology, Max Planck Institute for Human Development, Berlin, 14195, Germany, ${ }^{2}$ Institute of Cognitive Neuroscience, \\ Ruhr-Universität Bochum, Bochum, 44780, Germany, ${ }^{3}$ Brain Imaging Centre, Research Centre for Natural Sciences, Budapest, 1117, Hungary, and \\ ${ }^{4}$ Faculty of Education and Psychology, Eötvös Loránd University, Budapest, 1053, Hungary
}

The distinctiveness of neural information representation is crucial for successful memory performance but declines with advancing age. Computational models implicate age-related neural dedifferentiation on the level of item representations, but previous studies mostly focused on age differences of categorical information representation in higher-order visual regions. In an age-comparative fMRI study, we combined univariate analyses and whole-brain searchlight pattern similarity analyses to elucidate age differences in neural distinctiveness at both category and item levels and their relation to memory. Thirty-five younger (18-27 years old) and 32 older (67-75 years old) women and men incidentally encoded images of faces and houses, followed by an old/new recognition memory task. During encoding, age-related neural dedifferentiation was shown as reduced category-selective processing in ventral visual cortex and impoverished item specificity in occipital regions. Importantly, successful subsequent memory performance built on high item stability, that is, high representational similarity between initial and repeated presentation of an item, which was greater in younger than older adults. Overall, we found that differences in representational distinctiveness coexist across representational levels and contribute to interindividual and intraindividual variability in memory success, with item specificity being the strongest contributor. Our results close an important gap in the literature, showing that older adults' neural representation of item-specific information in addition to categorical information is reduced compared with younger adults.

Key words: aging; episodic memory; fMRI; neural dedifferentation; neural distinctiveness; representational similarity

\section{Significance Statement}

A long-standing hypothesis links age-related cognitive decline to a loss of neural specificity. While previous evidence supports the notion of age-related neural dedifferentiation of category-level information in ventral visual cortex, whether or not age differences exist at the item level was a matter of debate. Here, we observed age group differences at both levels as well as associations between both categorical distinctiveness and item specificity to memory performance, with item specificity being the strongest contributor. Importantly, age differences in occipital item specificity were largely due to reduced item stability across repetitions in older adults. Our results suggest that age differences in neural representations can be observed across the entire cortical hierarchy and are not limited to category-level information.

Received Aug. 4, 2020; revised Jan. 12, 2021; accepted Feb. 10, 2021.

Author contributions: M.K. analyzed data; M.K. and V.R.S. wrote the first draft of the paper; M.K., V.R.S.,

A.K., M.W.-B., and M.C.S. edited the paper; V.R.S., M.W.-B., and M.C.S. designed research; V.R.S. and A.K. performed research; M.C.S. wrote the paper.

This work was conducted within the projects "Lifespan Age Differences in Memory Representations (LIME)" (PI: M.C.S.) and "Lifespan Rhythms of Memory and Cognition (RHYME)" (PI: M.W.-B.) at the Max Planck Institute for Human Development. M.K. was supported by German Academic Scholarship Foundation scholarship. V.R.S. was a fellow of the International Max Planck Research School on the Life Course. M.W.B. was supported by German Research Foundation (Deutsche Forschungsgemeinschaft; WE 4269/2-1 and WE 4269/5-1) and Jacobs Foundation Early Career Research Fellowship. M.C.S. was supported by the MINERVA program of the Max Planck Society. We thank all student assistants who helped with data collection; Gabriele Faust and members of the LIME and RHYME projects for helpful feedback; and all study participants for their time.

*M.K. and V.R.S. contributed equally to this work.

${ }^{\dagger}$ M.W.-B. and M.C.S. contributed equally to this work as senior authors.

The authors declare no competing financial interests.

Correspondence should be addressed to Myriam C. Sander at sander@mpib-berlin.mpg.de or Markus Werkle-Bergner at werkle@mpib-berlin.mpg.de.

https://doi.org/10.1523/JNEUROSCI.2038-20.2021

Copyright $\odot 2021$ the authors

\section{Introduction}

A prominent theory of cognitive aging assumes that neural representations become less distinctive in old age and that this socalled neural dedifferentiation underlies age-related cognitive impairments (e.g., S. C. Li et al., 2001; J. Park et al., 2010; Koen and Rugg, 2019). In computational models, age differences have been ascribed to deficient neuromodulation that causes haphazard activation to identical informational input, leading to lower stability (i.e., consistency of neural activity across repetitions) of representations on the item level as well as increasing random activation variability within the network, leading to a decrease in the distinctiveness of the network's representations (S. C. Li et al., 2000, 2001; S. C. Li and Sikström, 2002). Importantly, deficient neuromodulation thus results in age differences in neural information representation that coexist at various levels, that is, 


\section{This article has been retracted}

at the level of item representations and the level of category representations, and jointly affect memory performance. However, previous age-comparative studies (mainly using fMRI) have nearly exclusively focused on age-related dedifferentiation in categorical representations, neglecting age differences at the level of item representations that possibly occur already during earlier stages of visual processing. Hence, while long-standing observations from behavioral aging research suggest a close connection between age differences in sensory processing and decline in higher order cognition (e.g., Lindenberger and Baltes, 1994; Baltes and Lindenberger, 1997; Schneider and Pichora-Fuller, 2000; K. Z. Li and Lindenberger, 2002), the limited available evidence has not yet allowed to settle the debate whether or not age differences in neural distinctiveness already exist at the level of item representations (Koen and Rugg, 2019). We therefore set out to track age differences at different representational levels, ranging from item-level specificity to category selectivity, with the goal to delineate their unique and joint contribution to memory performance in younger and older adults.

The available evidence clearly supports the notion of age-related neural dedifferentiation of category information. Focusing on age differences in the representation of broader categories, such as faces, houses, or objects within the ventral visual cortex (VVC) (D. C. Park et al., 2004; Payer et al., 2006; Voss et al., 2008; J. Park et al., 2012; Koen et al., 2019; Srokova et al., 2020), previous studies made use of the preferential response of these regions to specific stimulus categories (Kanwisher et al., 1997; Epstein and Kanwisher, 1998; Grill-Spector and Malach, 2004). For example, using conventional univariate analyses, D. C. Park et al. (2004) demonstrated that, compared with young adults, older adults exhibited less category-selective BOLD responses to stimuli of faces, houses, chairs, and pseudowords, providing the first human evidence for age-related neural dedifferentiation in the VVC (see also Payer et al., 2006; J. Park et al., 2012 and, accounting for trialwise BOLD variability: Koen et al., 2019; Voss et al., 2008). Directly probing the (dis-)similarity of distributed, multivariate activation patterns, with representational similarity (compare Edelman, 1998; Kriegeskorte et al., 2008) or decoding techniques (compare Haynes and Rees, 2006; Mur et al., 2009), subsequent studies revealed that different stimulus categories were represented less distinctively in category-selective areas in the VVC in older compared with younger adults (J. Park et al., 2010; Carp et al., 2011; Koen et al., 2019; Trelle et al., 2019). A few age-comparative studies also investigated neural distinctiveness at the within category (Goh et al., 2010) or even item level (Trelle et al., 2019). Unfortunately, with few exceptions (StLaurent et al., 2014; Sommer et al., 2019), these studies focused on predefined category-specific regions. However, it is very likely that processing of item-specific representations is also supported by other brain regions than those showing category selectivity (see, e.g., Kriegeskorte et al., 2007). We therefore speculated that age differences in item specificity may have gone unnoticed in most studies with ROI approaches that focused on category-specific brain regions.

Importantly, assuming that an altered signal-to-noise ratio in neural information processing leads to more variable neural responses in old age (S. C. Li et al., 2001), item-level specificity in older adults may depend critically on representational item stability, that is, the consistency with which individual items are represented across several instances of encoding. However, while studies with young-adults-only samples have already revealed the importance of item stability (inside and outside of categoryselective areas including, for instance, occipital cortex) for memory performance (Xue et al., 2010; Ward et al., 2013), so far only one study (Zheng et al., 2018) compared item stability across repeated encoding between younger and older adults. They found that, independent of their distinctiveness from other representations, item stability in the visual cortex was reduced in older adults and was associated with lower memory performance compared with young adults. Moreover, item stability was an important contributor to memory performance as indicated by subsequent memory effects (compare Paller and Wagner, 2002). However, age differences were less clear regarding item specificity, a measure calculated similarly to item stability but corrected for similarity of items from the same category. This inconsistency provoked criticism regarding the validity of the results (see Koen and Rugg, 2019).

In sum, there is a clear tension between model-derived predictions for neural dedifferentiation and available empirical support. While many studies provided coherent evidence for neural dedifferentiation with age at the category level (for review, see Koen and Rugg, 2019; Koen et al., 2020), they did not directly test a central tenet of computational models of dedifferentiation (S. C. Li et al., 2000, 2001; S. C. Li and Sikström, 2002), namely, reduced distinctiveness of single-item representations. Thus, whether age differences exist at the level of item representations is an ongoing debate in the field based on highly limited evidence (see e.g., Koen and Rugg, 2019). Hence, in the current age-comparative fMRI study, we combined conventional ROI-based univariate analysis and whole-brain searchlight pattern similarity analysis to elucidate age-related differences in neural specificity both at the level of categorical information as well as at the level of the individual items, and their relation to memory performance.

\section{Materials and Methods}

Participants

The face-house task described in the current report was embedded in a larger study with an overall sample consisting of 47 younger and 49 older healthy adults who participated in several structural MRI scans and additional behavioral tasks inside and outside the MR scanner. Thirty-nine young and 37 older adults completed the face-house task. We excluded 3 participants ( 2 young adults and 1 older adult) because of memory performance below chance level and 6 participants ( 2 young adults and 4 older adults) because of a failure to detect category-selective clusters (see below). Therefore, final analyses were based on a sample of 35 young adults (age: mean $=22.11$ years, $S D=2.70$ years, range $=18-27$ years; 17 female; 18 male) and 32 older adults (age: mean $=70.72$ years, $S D=2.26$ years, range $=67-75$ years; 19 female; 13 male) with normal or corrected-to-normal vision and no history of neurologic or psychiatric diseases. No participant was taking any medications known to affect brain functions. A potential confounding factor of age differences in fMRIbased measures are age-related changes in the neurovascular system (compare D'Esposito et al., 1999; Grinband et al., 2017; Lu and Liu, 2017; West et al., 2019). In the present study, participants were carefully screened for comorbidities, and their medications that could affect blood flow (e.g., hypertension) (compare Gazzaley and D'Esposito, 2005). Furthermore, we screened all older adults with the Mini-Mental State Examination (Folstein et al., 1975), and none scored below the threshold of 26 points $($ mean $=29.19 ; \mathrm{SD}=1.12$; range $=26-30)$. All participants gave written informed consent to take part in the experiment. All procedures were approved by the ethics committee of the German Society for Psychological Research.

\section{Stimuli and procedure}

Stimulus material. Overall, the stimulus set comprised 300 grayscale pictures from three different categories: 120 neutral faces (adapted from the FACES database; Ebner et al., 2010); 120 houses (in part adapted from D. C. Park et al., 2004; and in part obtained through web search); 
A Paradigm

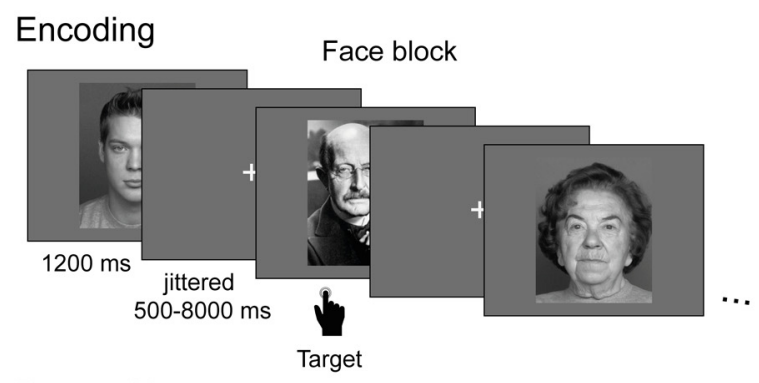

Recognition

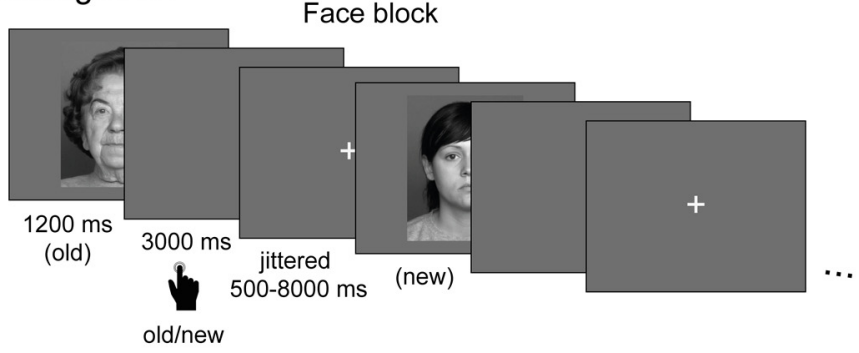

C Representational similarity levels

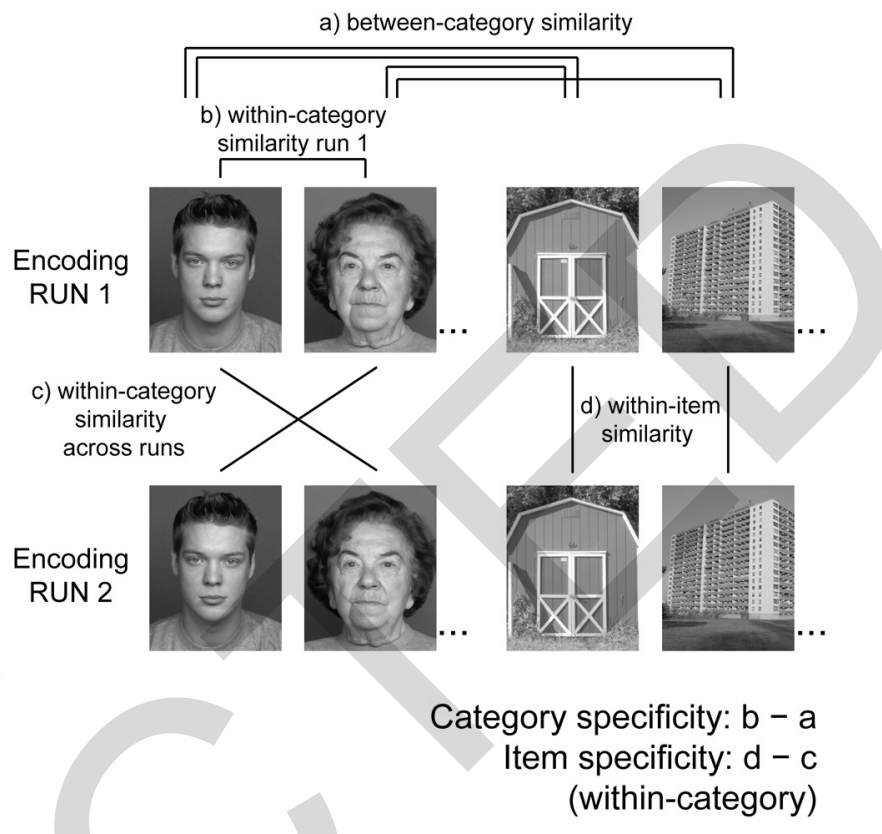

B Block design 20 exemplars +1 target

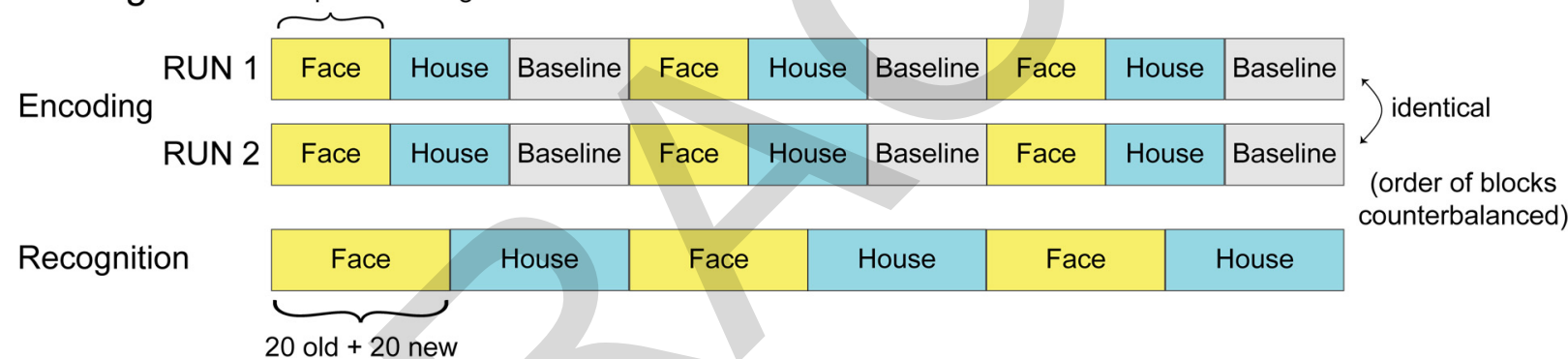

Figure 1. Task design and schematic of representational similarity levels. $\boldsymbol{A}$, The paradigm comprised an encoding (top) and recognition (bottom) task. During encoding, participants were asked to press a button whenever a previously trained target image appeared. During recognition, participants responded via button press whether each presented image was old or new. Here, only sample stimuli from a face block are shown for illustration, but also houses and baseline control stimuli were presented. $\boldsymbol{B}$, Illustration of the block design of encoding and recognition tasks. The encoding task consisted of two identical runs, each of which comprised 3 face, 3 house, and 3 baseline (phase-scrambled faces and houses) blocks in alternating and counterbalanced order (either starting with a face or house block). In each block, 20 exemplars of the respective category (face, house, baseline) were presented plus one target stimulus. The recognition task consisted of 3 face and 3 house blocks in alternating and counterbalanced order. In each block, 20 old and 20 new faces/houses were presented. C, Simplified illustration of the computed representational similarity levels. For each item presented in encoding run 1, neural pattern similarity was computed as the Pearson correlation of the BOLD pattern elicited by that item and the BOLD pattern(s) elicited by $(\mathbf{C} \boldsymbol{a})$ all items of the respective other category in run 1 (between-category similarity), $(\boldsymbol{C} \boldsymbol{b})$ all other items of the same category in run 1 (within-category similarity in run 1), (CC) all other items of the same category in run 2 (within-category similarity across runs), and (Cd) the same item in run 2 (within-item similarity; stability). For each subject, the respective similarity measure was averaged across items, resulting in one similarity value for each representational level. Category representation specificity was calculated as the difference of between-category and within-category similarity $(\boldsymbol{C} \boldsymbol{b}-\boldsymbol{C a})$. Item representation specificity was calculated as the difference of within-category (across runs) and within-item similarity $(\boldsymbol{C d}-\boldsymbol{C} \boldsymbol{c})$ for faces and houses, respectively. These similarities were assessed based on ROls as well as a searchlight approach.

and 60 phase-scrambled images (30 faces and 30 houses, constructed from randomly chosen pictures of the faces/houses sets) as control stimuli. Face stimuli furthermore consisted of four subcategories, split by age group (i.e., young vs old) and gender (i.e., female vs male). Similarly, house stimuli comprised four subcategories, namely, apartment buildings, family houses, sheds, and warehouses. In addition, three target stimuli were used for the encoding task, comprising a specific face image, a specific house image, and a phase-scrambled image with a white square in the center. All 240 nontarget face and house stimuli were split into two sets of 120 items (60 faces and 60 houses comprising 15 stimuli of each subcategory) to present one set during encoding and recognition (old items) and the other set only during recognition (new items). The same item sets were used as old or new items across subjects.

Procedure. Here, we focused on the face-house task consisting of an incidental encoding phase and a surprising recognition test, both conducted inside the MR scanner (see Fig. $1 A$ ). After verbal and written instructions, giving consent, and performing several behavioral tasks, including a short training of the encoding task, participants were positioned into the scanner. Here, they again practiced the task for a few trials. The encoding phase consisted of two identical runs, including 9 stimulus blocks each (see Fig. 1B). Stimuli were presented using Psychtoolbox (Psychophysics Toolbox) for MATLAB (The MathWorks). During each block, one target (for which participants had to respond) and 20 nontarget images of the same category (i.e., faces, houses, or phase-scrambled controls) were presented for $1200 \mathrm{~ms}$ with a fixation cross shown between trials (jittered; ranging from 500 to $8000 \mathrm{~ms}$ ). While face or house blocks included five images from each of the four subcategories, phase-scrambled blocks comprised 10 scrambled face images and 10 scrambled house images. Stimuli were randomly distributed into the blocks. Stimulus order was pseudo-randomized with the restriction that no subcategory appeared twice in a row and the target image was presented neither in the first four nor the last four trials of a block. The order of the blocks was alternating and counterbalanced across participants with either starting with a face or house block. Because of a technical failure, the identical stimulus order 
solution was used for all participants starting with a face block and in 36 participants starting with a house block. To ensure attention to all stimuli during encoding, subjects were instructed to engage in a vigilance task asking them to press a button with their right index finger whenever one of the three target images was presented. Such target trials were excluded from further analyses. In the second run, blocks and trials were exactly repeated as in the first run (i.e., each image was presented twice during encoding). In total, the encoding task lasted $22 \mathrm{~min}$.

After encoding, several structural scans were conducted (see below). Then, subjects had a break outside the scanner in which the surprise recognition test was explained to them before going back into the scanner to perform the recognition test. The duration between the encoding and recognition tasks was $\sim 30 \mathrm{~min}$. The recognition test consisted of three face and three house blocks presented in alternated and counterbalanced order. Each block contained 20 previously seen and 20 new images. Subjects were instructed to indicate whether a presented image was old or new via button press during stimulus presentation $(1200 \mathrm{~ms})$ or the following gray screen $(3000 \mathrm{~ms})$. The order of the trials was pseudorandomized with the restriction that images of the same subcategory or four old or new images did not appear successively. Furthermore, trials were separated by a jittered fixation cross (500-8000 ms). Again, because of a technical issue, an identical stimulus order was used for 13 participants starting with a face block and for 14 participants beginning with a house block. The recognition task lasted $\sim 26 \mathrm{~min}$.

\section{Behavioral data analyses}

Behavioral data were analyzed using custom-written MATLAB scripts. For key resources please see Table 1. Performance in the target detection task during encoding was measured as percentage of correct responses to targets. Memory performance in the recognition task was assessed by calculating the discrimination index $\operatorname{Pr}$ (Snodgrass and Corwin, 1988), which is an unbiased parameter for recognition performance. $P r$ was calculated as the difference between the hit rate (proportion of old responses to old images) and the false alarm rate (proportion of old responses to new images). We used $t$ tests to probe whether recognition performance was different from chance level, and whether memory performance differed for face and house images. Age differences in memory performance were assessed by computing an independent-samples $t$ test between younger and older adults. For effect sizes, we report Cohen's $d$.

\section{fMRI data collection and preprocessing}

All MR images were acquired with a 3T Siemens Magnetom Tim Trio scanner. MRI data acquisition included a T1-weighted structural image (MP-RAGE sequence: $\mathrm{TR}=2.5 \mathrm{~ms}, \mathrm{TE}=4.77$, flip angle $=7^{\circ}, \mathrm{TI}=1.1$ $\mathrm{ms}$, voxel size $\left.=1 \times 1 \times 1 \mathrm{~mm}^{3}\right)$ and $\mathrm{T} 2 *$-weighted $\mathrm{EPI}(\mathrm{TR}=2 \mathrm{~s}, \mathrm{TE}=$ $30 \mathrm{~ms}$, pixel size $=3 \times 3 \mathrm{~mm}^{2}$ ) sensitive to BOLD contrast. EPIs were acquired in two separated encoding and retrieval sessions, which each comprised 270 volumes with 36 axial slices of $3 \mathrm{~mm}$ thickness, separated by $3.3 \mathrm{~mm}$. As part of the larger study context, we additionally acquired turbo spin-echo proton density images, diffusion tensor images, and fluid attenuation inversion recovery.

MRI data were first organized according to "Brain Imaging Data Structure" specifications (Gorgolewski et al., 2016). Data processing was performed using the FMRIPrep toolbox (1.4.0) (Esteban et al., 2019) with the default processing steps incorporating the software packages: FSL, FreeSurfer, ANTs, and AFNI. First, each T1-weighted volume was corrected for intensity nonuniformity and skull-stripped to reconstruct brain surfaces. Brain-extracted T1-weighted images were spatially normalized to the ICBM 152 Nonlinear Asymmetrical template version 2009 c through nonlinear registration. Brain tissue segmentation of CSF, white matter, and gray matter was performed on the brain-extracted T1weighted image. Functional data were slice time-corrected, motion-corrected and coregistered to the normalized T1-weighted template (for further details, including software packages for each preprocessing step in FMRIPrep, see the online documentation under https://fmriprep.org/en/ stable/).

For univariate analyses, functional images were resampled to $4 \mathrm{~mm}$ isotropic voxels and spatially smoothed with a kernel of $4 \mathrm{~mm}$ FWHM. We computed a voxelwise GLM, including the first and second encoding run using SPM12. BOLD signal changes were modeled using separate regressors for face, house, and phase-scrambled control image blocks and 6 nuisance motion regressors, which were all convolved with a canonical HRF.

Pattern similarity analyses were conducted on functional images resampled to $2 \mathrm{~mm}$ isotropic voxels to enhance signal-to-noise ratio (Dimsdale-Zucker and Ranganath, 2018) and were based on single trial $\beta$ weights, including one trial-specific regressor, one regressor for all other trials, six motion regressors, and one run specific mean (Mumford et al., 2012). Trial specific regressors were modeled as a $1.2 \mathrm{~s}$ duration boxcar function convolved with a canonical HRF.

\section{Defining ROIs}

We used a cluster-based approach to define individual functional ROIs most strongly engaged by processing faces or houses during both encoding runs. To restrict our analyses to category-selective processing areas, we defined an anatomic mask of the VVC using the automated anatomic labeling atlas, including bilateral fusiform gyrus, parahippocampal gyrus, and the inferior temporal gyrus (D. C. Park et al., 2004). Within this mask, we then contrasted BOLD responses to face versus phasescrambled control image blocks and BOLD responses to house versus phase-scrambled control image blocks for each subject. All adjacent voxels exceeding the uncorrected threshold of $p<0.005$ were defined as one cluster. As mentioned above, 2 young and 4 older participants were excluded because clusters included $<10$ voxels. The face-selective ROI was defined as the cluster with the highest averaged $t$ value for faces compared with phase-scrambled images and the house-selective ROI as the cluster with the highest averaged $t$ value for houses compared with phase-scrambled images leading to the formation of two individual functional ROIs. We defined no cutoff of cluster size across subjects. The average number of voxels in the face- and house-selective clusters did not differ between young and older adults (face-selective ROIs: $M_{\text {young }}=$ $78.8 ; M_{\text {old }}=82.3 ; t_{(65)}=-0.24 p=0.810$; house-selective ROIs: $M_{\text {young }}=$ $\left.70.7 ; M_{\text {old }}=85.2 ; t_{(65)}=-1.11 p=0.273\right)$. Within participants, on average only $4.15 \%$ (fusiform gyrus: $4.74 \%$; parahippocampal gyrus: $1.64 \%$; inferior temporal gyrus: $2.40 \%$ ) of all voxels of the face- and house-selective ROIs overlapped, underlining separated category-selective clusters in the VVC.

\section{ROI analysis of category-selective processing}

To estimate the fMRI response magnitudes evoked by face and house images within each individual ROI, we first contrasted the BOLD signal elicited by face and house image blocks against the BOLD signal of phase-scrambled control image blocks within the first encoding run. Then, we averaged all $t$ values within each ROI to obtain a standardized response value to faces or houses, respectively. Category selectivity scores were computed by contrasting standardized BOLD responses to the preferred versus nonpreferred image category within each individual ROI, for example, BOLD response to faces versus BOLD response to houses in the face-selective ROI. To analyze selectivity scores on group level, we used a 2 (Age Group) $\times 2$ (ROI) mixed factorial ANOVA.

Category selectivity scores were collapsed across ROIs to calculate mean selectivity scores within each participant. This category selectivity score index was correlated with $\mathrm{Pr}$ across all participants using Pearson correlation. Furthermore, we conducted partial correlations between category selectivity scores and $\mathrm{Pr}$ with age group as control variable to test whether this relationship is independent of age.

\section{ROI analysis of category representation specificity}

To estimate the specificity of neural category representations within category-selective clusters, we compared the within-category similarity to the between-category similarity within the first run using pattern similarity analysis (Kriegeskorte et al., 2008) (see Fig. 1C). Similarity indices were based on Fisher $z$-transformed Pearson correlations between single-trial $\beta$ weights calculated across voxels. Within- and between-category similarity was calculated separately in both ROIs. Within-category similarity was only calculated for items from the preferred category of the selected area (Koen et al., 2019) and was only computed for items from different blocks to control for potential confounds because of time- 
Table 1. Key resources

\begin{tabular}{lll}
\hline Resource & Source & Identifier \\
\hline Software and algorithms & & \\
FieldTrip Toolbox & http://www.FieldTriptoolbox.org & RRID:SCR_004849 \\
MATLAB 2020a & http://www.mathworks.com & RRID:SCR_001622 \\
Psychtoolbox & http://psychtoolbox.org & RRID:SCR_002881 \\
SPM12 & https://www.fil.ion.ucl.ac.uk/spm & RRID:SCR_007037 \\
FMRIPrep & http://fmriprep.readthedocs.io & RRID:SCR_016216 \\
\hline
\end{tabular}

dependent correlations in hemodynamic responses (Dimsdale-Zucker and Ranganath, 2018). For each item, the within-category similarity was computed as the average across-voxel Pearson correlation of the BOLD activity pattern of such item to all other items from the same preferred category in a category-selective area (e.g., similarity of a face trial to all other face trials in the face area). For each subject, we averaged within-category similarity across items, resulting in one within-category similarity value for each ROI. The between-category similarity of each item was calculated as the across-voxel Pearson correlation of the BOLD activity pattern of the item to the BOLD activity pattern of all items from the other category (e.g., similarity of a face trial to all house trials in the face area). We again averaged between-category similarity across items to estimate one between-category similarity value for each ROI. To test for differences in pattern similarity values, we used a 2 (Age Group) $\times 2$ (within/between-category similarity) $\times 2$ (ROI) mixed factorial ANOVA. To link category specificity and memory performance, we collapsed category specificity across ROIs and calculated zero-order and partial Pearson correlations between category specificity and $\operatorname{Pr}$ controlled for age.

\section{Searchlight analyses of category representation specificity}

We conducted separated searchlight analyses for faces and houses. For each voxel in the brain, we compiled the difference of within- and between-category similarity (i.e., category specificity) within an 8-mmradius sphere centered on that voxel using modified scripts of the MATLAB Toolbox for representational similarity analysis (Nili et al., 2014). Within- and between-category similarity was calculated as described in the previous section. Thus, we derived a whole-brain map for each subject where each value indicated the category specificity of the according sphere.

We used nonparametric, cluster-based, random permutation statistics adapted from the FieldTrip toolbox (Oostenveld et al., 2011) to test for age differences in category specificity across the brain. First, independent $t$ tests were conducted comparing category specificity of young and older adults for each voxel. Adjacent voxels exceeding the defined threshold of $p<0.005$ were grouped into clusters. The sum of all $t$ values of these empirical clusters was determined as the respective test statistic. We used the Monte Carlo method to compute a reference distribution for the summed cluster $t$ values. Category specificity of both age groups was randomly assigned (1000 permutations) into two new category specificity sets. In every permutation, an independent $t$ test was conducted between the newly generated random sets within each cluster. The sum of these $t$ values during each permutation was included in the estimation of the reference distribution of a cluster.

Searchlight analyses of item representation specificity

To investigate the specificity of item representations, we compared within-item similarity to between-item similarity (i.e., item specificity) using pattern similarity searchlight analysis (see Fig. 1C). Matching the category specificity searchlight analysis, we compiled the within- minus between-item similarity for each voxel within an 8-mm-radius sphere. For each item, within-item similarity was calculated as the across-voxel Pearson correlation of the BOLD activity pattern of a given item from the first encoding run to the BOLD activity pattern of the same item from the second encoding run in each sphere. Between-item similarity was computed as the correlation of the BOLD activity pattern of an item of the first encoding run to the BOLD activity pattern of all other items from the same category of the second encoding run. For each participant, we then averaged within- and between-item similarity across items. We used the resulting within- and between-item similarity brain maps of each participant to calculate one item specificity brain map for each subject. To test for age differences in item specificity across the brain, we used nonparametric, cluster-based, random permutation statistics (see Searchlight analysis of category representation specificity). First-level statistics compared item specificity of young and older adults for each voxel. Second-level statistics compared the summed $t$ value of the empirical clusters to a reference distribution computed using the Monte Carlo procedure.

Furthermore, we averaged item specificity for faces and houses for each participant to estimate one item specificity score per participant. Respective item specificity scores were correlated with $\mathrm{Pr}$ across participants using Pearson correlations and partial correlations to control for age group differences.

All age comparisons of neural selectivity (see above) and specificity are based on within-person differences (e.g., the difference between responses to preferred and nonpreferred image categories), which reduces the impact of potential confounding factors in the neurovascular system as opposed to comparing absolute measures, such as absolute BOLD activation between age groups (compare Rugg and Morcom, 2005). Crucially, the interaction with memory performance supports the interpretation that the identified age differences are indeed related to neurocognitive functions and not merely confounded by unspecific neurovascular differences.

Analyzing the relationship between differentiation on multiple representational levels and memory performance using linear mixed models

(LMMs)

We used LMM to examine the contribution of our distinctiveness measures (category selectivity [Csel], category specificity [Cspec], and item specificity [Ispec]) to memory success (Pr). In order to remove main effect differences between the groups, we standardized (with a mean of zero and unit SD) all variables within the respective age group. In order to test whether any association between the distinctiveness measures and memory success were dependent on age group membership, we also tested for the respective interactions. In addition, we allowed for a random intercept for each participant (ID). In sum, using the fitglme function in MATLAB, we fit the following random intercept model (in Wilkinson notation, compare Wilkinson and Rogers, 1973):

$$
\begin{aligned}
& P r \sim 1+C s e l+C s p e c+I s p e c+C s e l: \text { agegroup }+ \text { Cspec } \\
& \quad: \text { agegroup }+ \text { Ispec }: \text { agegroup }+(1 \mid I D)
\end{aligned}
$$

Disentangling the effects of age and memory performance on item specificity

We aimed to disentangle whether lower item representation specificity in older adults occurred because of lower item stability or higher between-item similarity in older adults compared with younger adults and which of these two factors was related to memory performance. Therefore, we averaged within- and between-item similarity for later remembered and forgotten items separately in the significant face and house clusters of the conducted searchlight analyses. To test for differences in item similarity, we conducted a 2 (Age Group) $\times 2$ (within-item/ within-category similarity) $\times 2$ (Memory: remembered/forgotten) $\times 2$ (ROI) mixed factorial ANOVA.

Furthermore, we examined item stability at the trial level by computing a generalized LMM (GLM) with binary memory response outcome (hit or miss) as dependent variable, trialwise item stability, that is, within-item similarity (WI), between-item similarity (BI), age group, the respective interactions with age group, and the individual response bias $(\mathrm{Br})$ calculated as false alarm rate/(1 - Pr) (Corwin, 1994) as fixed effects, and subject ID as random effect (intercept). We used a binomial distribution with a logistic link function using restricted maximum likelihood estimation to fit the data as follows: 
3504 • J. Neurosci., April 14, 2021 • 41(15):3499-3511

memory response $\sim 1+W I+B I+$ agegroup $+B r$

$+W I$ : agegroup $+B I$ : agegroup $+(1 \mid I D)$

Resource availability

Data and code for all analyses of this manuscript are publicly available on osf: https://osf.io/t8dpv/.

\section{Results}

\section{Behavioral results}

Healthy young adults $(\mathrm{n}=35$, age: mean $=22.11$ years, $\mathrm{SD}=2.70$ years, range $=18-27$ years $)$ and older adults $(n=32$, age: mean $=70.72$ years, $S D=2.26$ years, range $=67-75$ years ) incidentally encoded images of faces and houses, followed by a surprise recognition test (Fig. 1A). To ensure adequate processing of the presented face and house images, participants performed a low-level vigilance task during encoding (i.e., pressing a button whenever an interspersed predefined target image appeared). Both younger and older participants performed near ceiling in the vigilance task (percentage of correct answers: $M_{\text {young }}=$ $98.95 \%, S D_{\text {young }}=4.69 \% M_{\text {old }}=98.77 \%, S D_{\text {old }}=3.54 \%$ ), reflecting high engagement with the encoding task. Memory performance in the recognition task was assessed by the discrimination index $\operatorname{Pr}$ calculated as the difference between the hit rate and false alarm rate, which can range from -1 to 1 with 0 representing chance level. In both age groups, the mean memory performance exceeded chance level $\left(\operatorname{Pr}_{\text {young }}=0.24, t_{\text {young }}(34)=12.18\right.$, $p_{\text {young }}<0.001, d_{\text {young }}=2.06, \operatorname{Pr}_{\text {old }}=0.19, t_{\text {old }}(31)=9.16, p_{\text {old }}<$ $0.001, d_{\text {old }}=1.62$ ) and did not differ between face and house images (faces: $P r_{\text {young }}=0.23$, houses: $P r_{\text {young }}=0.25$, $t_{\text {young }}(35)=0.93, p_{\text {young }}=0.358$, faces: $P r_{\text {old }}=0.17$, houses: $\left.P r_{\text {old }}=0.21, t_{\text {old }}(31)=1.67, p_{\text {old }}=0.105\right)$. Older adults gave more "old" responses for both previously learned (hit rate young $=0.50$, hit rate $\left._{\text {old }}=0.61, t_{(65)}=-3.08, p=0.003, d=0.75\right)$ and completely new images compared with younger adults (false alarm rate young $_{1}=0.26$; false alarm rate $_{\text {old }}=0.41 ; t_{(65)}=-4.92$, $p<0.001, d=1.34)$. An independent-samples $t$ test revealed no significant difference in memory performance $(P r)$ between age groups $\left(t_{(65)}=1.62, p=0.110, d=0.40\right)$.

Category-selective processing in the VVC differs between age groups and is related to memory performance

Age-related neural dedifferentiation has previously been described as less distinct recruitment of category-selective areas located in the VVC (D. C. Park et al., 2004). Within categoryselective functional ROIs (Fig. 2), we calculated a category selectivity score as the difference in BOLD responses to images of the preferred versus the nonpreferred category (e.g., the difference in BOLD responses to images of faces versus houses in the faceselective ROI). A 2 (Age Group) $\times 2$ (ROI) mixed factorial ANOVA revealed that older adults showed reduced categoryselective processing compared with young adults $\left(F_{(1,65)}=12.59\right.$, $p<0.001$; Fig. $3 A$ ). Furthermore, the magnitude of the selectivity score did not differ between ROIs $\left(F_{(1,65)}=0.22, p=0.643\right)$, and there was no interaction between ROI and age $\left(F_{(1,65)}=0.06\right.$, $p=0.813$ ). Thus, replicating previous observations (D. C. Park et al., 2004; Koen et al., 2019), our findings emphasize that older adults engage category-selective areas in the VVC less distinctively compared with younger adults when encoding visual information.

If neural dedifferentiation occurred because of age-related increases of haphazard neural activation as claimed by S. C. Li et
Kobelt, Sommer et al. • Age Differences in Neural Distinctiveness

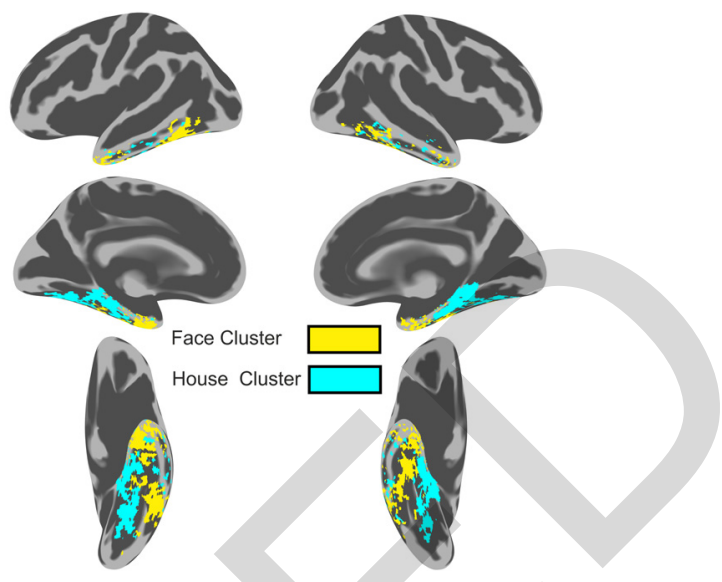

Figure 2. Identification of category-selective clusters in VVC. Surface rendered illustration of nonoverlapping category-selective ROls across all participants. Yellow represents faceselective ROI. Cyan represents house-selective ROI. Notably, the face-selective ROI is predominantly located in the lateral VVC and the house-selective ROI in the medial VVC closely resembling the category-selective areas observed by J. Park et al. (2012).

al. (2001), lower category selectivity should result from increased neural noise reflected in increased responses to images of the nonpreferred category in older adults (i.e., neural broadening) (J. Park et al., 2012). However, the current evidence is fairly mixed as there are studies supporting neural broadening and studies showing the opposite effect: reduced responses to images of the preferred category (i.e., neural attenuation; for review, see Koen and Rugg, 2019). To unravel whether age-related reductions in category-selective processing occurred because of (1) neural broadening, (2) neural attenuation, or (3) a mixture of both processes (see J. Park et al., 2012), we conducted post hoc $t$ tests and used Bonferroni correction to control for multiple comparisons (Fig. 3B). Across ROIs, older adults showed increased BOLD responses to images of the nonpreferred category compared with younger adults $\left(t_{(65)}=2.38, p=0.040\right)$, whereas BOLD responses to preferred images did not differ between age groups $\left(t_{(65)}=-1.13, p=0.526\right)$. Hence, the observed age-related neural dedifferentiation in category processing most likely resulted from an increased engagement of category-specific regions by nonpreferred stimuli in line with the theoretical assumption that broadening effects of the neural tuning curve underlie the loss of neural distinctiveness in older adults (S. C. Li et al., 2001).

To test whether interindividual variability in category-selective processing was related to memory performance in the recognition task, zero-order and partial correlations were computed across participants using Pearson's $r$. Selectivity scores were averaged across face- and house-selective ROIs in the VVC. Across participants, selectivity scores were related to memory performance $(r=0.34, p=0.005$; Fig. $3 C)$. Importantly, this correlation remained significant when controlling for age group using partial correlations $(r=0.29, p=0.019)$. Thus, dedifferentiated visual processing in category-selective areas, as primarily observed in older adults, was related to poorer memory performance independent of age.

\section{Age differences in category representation specificity are restricted to the VVC}

Since category selectivity as assessed with univariate methods relies on averaged BOLD signal changes and does not allow to quantify the distinctiveness of specific item representations, we next used pattern similarity analysis (Kriegeskorte et al., 2008) to 
A

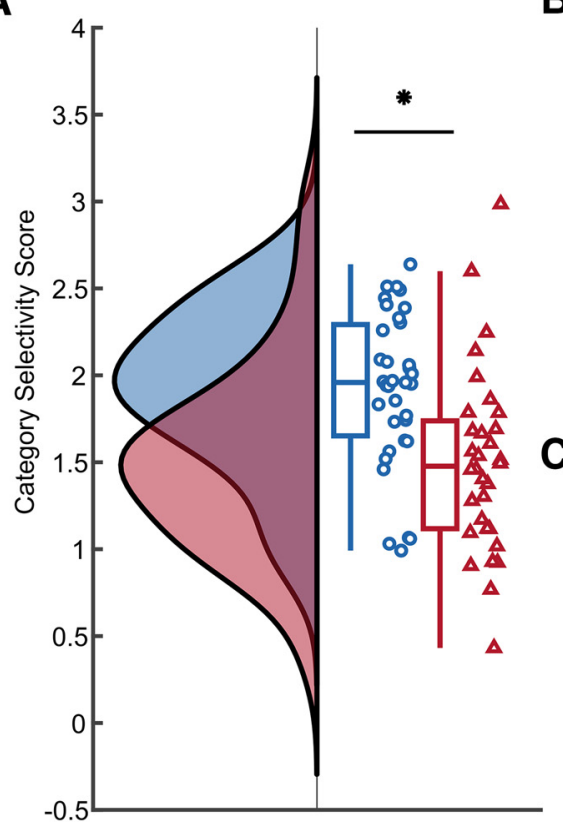

B

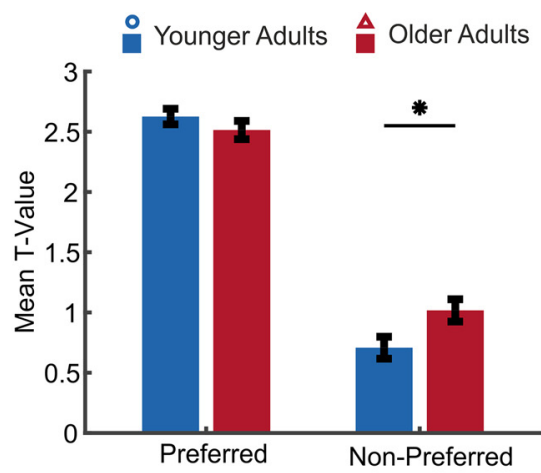

\section{}

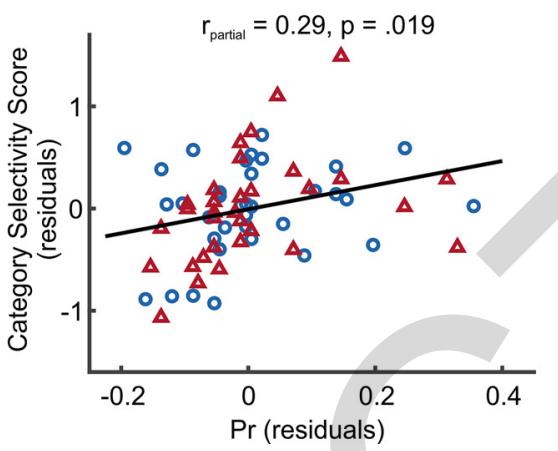

Figure 3. Age differences in category-selective processing. $\boldsymbol{A}$, Selectivity score for younger adults (blue; circles) and older adults (red; triangles). Group distributions shown as unmirrored violin plot and boxplots with medians and $95 \% \mathrm{Cl}$ with whiskers representing second and 98th percentiles (Allen et al., 2019). B, BOLD response to preferred and nonpreferred categories within functionally defined VVC ROls. The BOLD response is expressed as the averaged $t$ values for preferred/nonpreferred categories against phase-scrambled images within the respective ROls. Error bars indicate SEM. C, Scatterplot illustrating partial correlation between selectivity scores and corrected recognition $(\mathrm{Pr})$ controlling for age. Each circle/triangle represents an individual younger/older adult. For illustrative purposes, the best-fitting least-squares line is plotted. $*$ Significant group differences $(p<0.05)$.

A

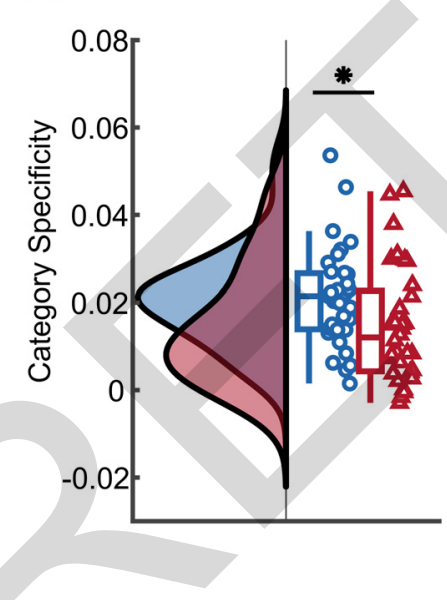

B
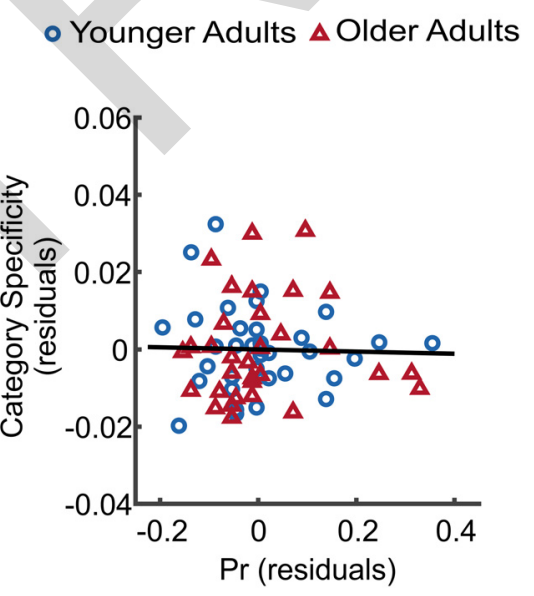

Figure 4. Category representation specificity in the VVC. $\boldsymbol{A}$, Category specificity calculated as the difference between within- and between-category similarity for younger adults (blue; circles) and older adults (red; triangles). Group distributions as unmirrored violin plot and boxplots with medians and 95\% Cl with whiskers representing second and 98th percentiles. $\boldsymbol{B}$, Partial Pearson correlation of category specificity and corrected recognition (Pr) controlling for age. For illustrative purposes, the best-fitting least-squares line is plotted. $*$ Significant group differences $(p<0.05)$.

investigate the categorical specificity of item representations. This analysis still focuses on differences on the categorical level but uses item-level information instead of averaged activation differences. Thus, we defined the specificity of neural category representations (category specificity) as the difference of within-category similarity and betweencategory similarity following an approach widely used in previous studies (Carp et al., 2011; Koen et al., 2019; Trelle et al., 2019).

A 2 (Age Group) $\times 2$ (within vs betweencategory similarity) $\times 2(\mathrm{ROI})$ mixed factorial ANOVA revealed that within-category similarity was higher than between-category similarity across age groups $\left(F_{(1,65)}=146.70\right.$, $p<0.001$; Fig. 4A). Moreover, the difference between within- and between-category similarity differed by Age Group $\left(F_{(1,65)}=4.85\right.$, $p=0.031$, indicating lower category specificity in older than younger adults. Neither the main effects of age $\left(F_{(1,65)}=2.07, p=0.155\right)$ or ROI $\left(F_{(1,65)}=0.05, p=0.820\right)$ nor any further interactions reached significance $(p$ values $>0.268$ ).

In contrast to most previous studies, which restricted their analyses on category-selective areas in the VVC, we used a searchlight approach to explore age differences in category specificity across the whole brain (Nili et al., 2014). In contrast to the ROI analysis, wholebrain analyses revealed no group differences in category specificity for houses $(p>0.336)$ and furthermore indicated that older adults showed higher category specificity for faces in the superior temporal gyrus $(p=0.006)$. Thus, while the ROI-based multivariate approach provided evidence supporting age differences in category specificity as reported by several previous studies (Carp et al., 2011; Koen et al., 2019; Trelle et al., 2019), the whole-brain approach did not. This result indicates that an agerelated decline for category information may be restricted to category-specific brain regions and less pronounced than thought, such that it does not survive exploratory whole-brain analyses.

We also tested whether interindividual differences in the specificity of neural category representations in category-specific brain regions were related to memory performance across participants (Fig. $4 B$ ). Specificity of neural category representations was calculated as the difference of within- and between-category similarity averaged over face- and house-selective areas. Neural category representation specificity was not related to memory performance using zero-order $(r=0.03$, $p=0.834$ ) or partial Pearson correlations controlling for age $(r=-0.03, p=0.829)$. Thus, we found no evidence that the specificity of neural category representations was related to memory performance.

In addition to categories and individual items (see below), the presented stimuli can be subdivided into subcategories (e.g., 
young female faces, warehouses). We did not identify any significant associations between representational subcategory specificity and memory or age (but see Kuhl and Chun, 2014; Trelle et al., 2019).

\section{Item representation specificity in occipital regions is reduced in older adults and linked to memory performance}

In contrast to previous studies (Carp et al., 2011; Koen et al., 2019), our results only partially support lower category specificity in older adults (i.e., when restricting the analyses to category-specific ROIs). However, theoretical accounts based on computational models emphasize that age-related dedifferentiation is particularly reflected in reduced specificity of neural representations of individual items (S. C. Li et al., 2001), which are not restricted to category-selective areas but are found in large-scale neural networks (Xue, 2018). Therefore, we applied pattern similarity analyses across the whole brain to investigate age-related changes in neural item specificity.

Within each sphere, within-item similarity was calculated as the similarity of BOLD activity patterns across the two repetitions of a given item (i.e., item stability; Fig. 1C). We compared this item stability with the respective within-category similarity to capture the specificity of neural item representations (i.e., item specificity).

For faces, older adults showed smaller item specificity compared with younger adults in occipital regions $(p<0.001)$, including bilateral intracalcarine cortex, lateral occipital cortex, and occipital fusiform cortex (Fig. 5A). For houses, older adults also showed smaller item specificity than younger adults in occipital areas $(p=0.006)$ comprising bilateral lingual gyrus, intracalcarine cortex, and left occipital fusiform cortex (Fig. 5B). Thus, our findings underline that older adults form less specific item representations in occipital areas than younger adults.

We also tested whether interindividual differences in item specificity were related to overall memory performance (Fig. $5 C$ ). For this analysis, we averaged item specificity over both categories in their respective occipital clusters. Neural item representation specificity was related to memory performance using zero-order $(r=0.39, p=0.001)$ and partial Pearson correlations controlling for age $(r=0.35, p=0.004)$. Equivalently, item stability alone showed the same between-person association with performance (zero-order: $r=0.42, p<0.001$; partial: $r=0.38, p=0.002$ ).

Thus, high item representation specificity/stability accounts for interindividual differences in memory performance of younger and older adults.

\section{Linking neural dedifferentiation across representational levels in relation to memory performance}

Given the coexistence of age differences in category selectivity, category specificity, and item specificity and their differential associations with recognition performance, we used LMMs to examine their contribution to memory success and the interaction with age. We observed a significant association between memory success and item specificity (main effect: $t_{(60)}=2.31 ; p=0.024$ ). Neither the main effect of category selectivity $\left(t_{(60)}=1.45 ; p=0.15\right)$ nor the main effect of category specificity $\left(t_{(60)}=-1 ; p=0.32\right)$ reached significance. Crucially, none of the interaction effects reached significance (all $|t|<0.95$; all $p>0.34$ ), providing no evidence for differential associations between measures of distinctiveness and memory success across age groups.

To further investigate the association between distinctiveness at different representational levels, we correlated all measures with each other. All neural distinctiveness measures were related to each other across participants (Table 2). However, the correlations between item and category measures appear to be mostly driven by overall age differences: When controlling for age using partial correlations, only the relationship between category selectivity and category specificity remained significant $(r=0.37, p=0.003)$, whereas partial correlations of item specificity to category selectivity $(r=0.14, p=0.276)$ and category specificity $(r=0.17, p=0.176)$ were not significant. These results underline previous theoretical distinctions between distinctiveness measures on category and item level (Koen and Rugg, 2019; Koen et al., 2020) and furthermore emphasize the close relationship between both dedifferentiation measures on category level (Koen et al., 2019).

Age and performance differences in occipital item specificity are driven by item stability

Our LMM analysis revealed that differences in item specificity account for interindividual differences in memory performance over and above the variance explained by all variables combined. Importantly, if, as assumed by S. C. Li et al. (2001), more variable neural responses are at the core of age-related reductions in 
This article has been retracted

Table 2. Association between distinctiveness measures and memory performance (zero-order Pearson correlations $r$, with $p$ in parentheses)

\begin{tabular}{|c|c|c|c|c|c|}
\hline & (1) & $(2)$ & (3) & (4) & (5) \\
\hline (1) Memory performance & - & - & - & - & - \\
\hline (2) Category selectivity & $0.34(0.005)$ & - & - & - & - \\
\hline (3) Category specificity & $0.03(0.834)$ & $0.43(<0.001)$ & - & - & - \\
\hline (4) Item specificity & $0.39(0.001)$ & $0.35(0.003)$ & $0.29(0.016)$ & - & - \\
\hline (5) Item stability & $0.42(<0.001)$ & $0.36(0.002)$ & $0.34(0.005)$ & $0.99(<0.001)$ & - \\
\hline (6) Age group & $-0.20(0.110)$ & $-0.40(<0.001)$ & $-0.26(0.031)$ & $-0.64(<0.001)$ & $-0.63(<0.001)$ \\
\hline
\end{tabular}

neural specificity, item specificity in older adults depends critically on the consistency with which individual items are represented across several instances of encoding (i.e., item stability). To test whether lower item specificity in older adults was indeed because of lower item stability (within-item similarity) or higher between-item (within-category) similarity, compared with younger adults and whether specifically higher item stability was related to a higher probability of successful memory retrieval, we compared similarity indices in the observed occipital clusters with a 2 (Age Group) $\times 2$ (withinvs between-item similarity) $\times 2$ (Memory: remembered/ forgotten $) \times 2$ (ROI) mixed factorial ANOVA.

Younger adults showed higher similarity scores compared with older adults $\left(F_{(1,65)}=22.25 ; p<0.001\right)$ and within-item similarity was generally higher than between-item similarity $\left(F_{(1,65)}=435.87 ; p<0.001\right)$. In line with the searchlight analysis, the difference between within-item similarity and between-item similarity (i.e., item specificity) was smaller in older than in younger adults as indicated by a significant interaction of age and within- vs between-item similarity $\left(F_{(1,65)}=25.05 ; p<0.001\right.$; Fig. 6A). Post hoc tests revealed that within-item similarity (i.e., item stability) $\left(t_{(65)}=4.94, p<0.001\right)$ was lower in older adults compared with younger adults, whereas between-item similarity $\left(t_{(65)}=2.16, p=0.069\right)$ did not differ between age groups after multiple error correction. Within- and betweenitem similarity differences were not different across ROIs $\left(F_{(1,65)}=3.53 ; p=0.064\right)$.

Most crucially, subsequently remembered items showed higher similarity scores compared with subsequently forgotten items $\left(F_{(1,65)}=7.85, p=0.007\right)$, which interacted with within- vs between-item similarity $\left(F_{(1,65)}=17.51 ; p<0.001\right.$; Fig. $6 B$ ). Post hoc $t$ tests revealed that within-item similarity (i.e., item stability) was higher for subsequently remembered than forgotten items $\left(t_{(66)}=3.90, p<0.001\right)$, while there was no difference in between-item similarity $\left(t_{(66)}=-1.17\right.$, $p=0.248)$. We found no interaction between memory and age group $\left(F_{(1,65)}=3.90 ; p=0.052\right)$ or other significant effects ( $p$ values $>0.316)$.

In addition to the ANOVA computed above, which contrasts the average representational similarities of later remembered and forgotten stimuli and identifies a critical role of item stability, we also ran a generalized LMM (GLM) to examine trialwise variability in item stability and how it predicts memory outcome (Fig. $6 C$ ). We tested whether binary memory response outcome (hit or miss) could be predicted by trialwise item stability, that is, within-item similarity (WI), between-item similarity (BI), age group, the respective interactions with age group, and the individual response bias $(\mathrm{Br})$. In line with the previously applied mixed factorial ANOVA (see above), we found that trialwise item stability predicted memory outcome $(\log$ odds $=0.12,95 \%$ CI $[0.06,0.18])$ in that trials showing larger item stability during encoding had a higher probability to be correctly recognized later on. Furthermore, there was a significant effect of response bias $(\log$ odds $=3.50,95 \%$ CI $[2.94,4.05])$ showing that participants with a bias to respond "old" showed more hits compared with misses. In contrast, neither between-item pattern similarity, age group, nor any of the interactions reached significance (Table 3 ).

Thus, item stability was lower in older than in younger adults and supported subsequent recognition performance on a trialwise level emphasizing the role of less stable item representations for age-related memory decline.

\section{Discussion}

The present fMRI study investigated age differences in the distinctiveness of neural information representations supporting episodic memory performance. We provided a comprehensive account of age differences at different representational levels ranging from category selectivity to item specificity and item stability and delineated their contribution to memory performance in younger and older adults. Combining conventional ROI-based univariate analysis and whole-brain multivariate pattern similarity analysis, we demonstrated that age differences in neural distinctiveness coexist at various representational levels, and take the form of reduced category-selective processing in VVC regions, and reduced specificity of item representations in occipital areas. The study revealed the contribution of both categorical distinctiveness and item specificity to memory performance, with item specificity being the strongest contributor to it. Age differences in occipital item specificity were largely due to reduced item stability across repetitions in older adults, and higher item stability was related to subsequent memory success. Whether or not age differences exist at the item level was a matter of debate that could so far not be settled because of limited evidence (Koen and Rugg, 2019). Our results close this gap in the literature by demonstrating that older adults' neural representation of item-specific information in addition to categorical information is indeed reduced compared with younger adults.

\section{Age-related neural dedifferentiation coexists at different representational levels}

Previous fMRI studies investigating neural dedifferentiation focused on broader concept representations by assessing univariate category-selective processing (e.g., D. C. Park et al., 2004) and/or multivariate category-specific representations (e.g., Carp et al., 2011), mainly in regions of the VVC. In the current study, ROI-based analyses within category-specific ROIs replicated previous findings: Univariate analyses revealed age-related neural dedifferentiation in terms of reduced category-selective processing (e.g., D. C. Park et al., 2004), and multivariate pattern similarity analyses revealed reduced categorical specificity (e.g., Carp et al., 2011; Koen et al., 2019) in older compared with younger adults. As predicted by computational models (S. C. Li et al., 2000, 2001; S. C. Li and Sikström, 2002), age differences in neural category differentiation resulted from neural broadening, that is, increased activation to nonpreferred stimuli in the older adults, with no age differences in activation differences for preferred 


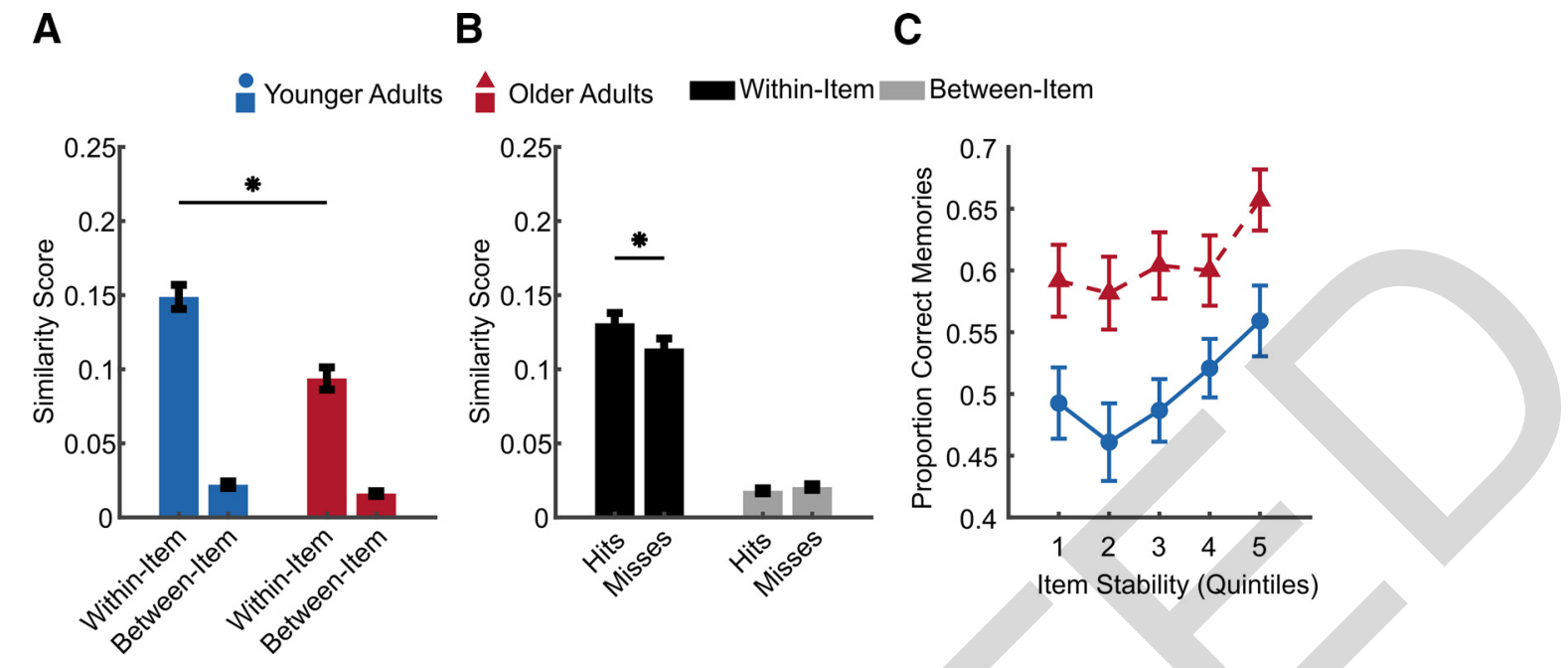

C

Figure 6. Item representation specificity and memory performance. $\boldsymbol{A}$, Within-item and between-item similarity in both age groups indicates that lower item specificity in older adults is mostly driven by lower item stability. $\boldsymbol{B}$, Comparing within- and between-item similarity for subsequently remembered items (hits) and not-remembered items (misses) across age groups shows that item stability was higher for later remembered than not-remembered items independent of age. $\boldsymbol{C}$, Trialwise item stability is linked to an increased probability of correct memories. For visualization, memory outcome was binned into quintiles according to trialwise item stability in each subject. Within each age group, the proportion of correct memories was averaged across participants. Error bars indicate SEM. *Significant group differences $(p<0.001)$.

Table 3. Results for GLM: memory response $\sim 1+\mathrm{WI}+\mathrm{BI}+$ agegroup + $\mathrm{Br}+$ Wl:agegroup + Bl:agegroup $+(1|| \mathrm{D})$

\begin{tabular}{lcrrrr}
\hline Fixed effects predictors & Log odds & \multicolumn{1}{l}{$p$} & & $95 \%$ Cl lower & $95 \%$ Cl upper \\
\hline Item stability (WI) & 0.12 & 3.74 & $<0.001$ & 0.06 & 0.18 \\
Between-item similarity (BI) & -0.02 & -0.66 & 0.508 & -0.08 & 0.04 \\
Response bias (Br) & 3.50 & 12.39 & $<0.001$ & 2.94 & 4.05 \\
Age group & -0.14 & -1.49 & 0.136 & -0.31 & 0.04 \\
WI $\times$ age group & -0.001 & -0.03 & 0.975 & -0.09 & 0.09 \\
BI $\times$ age group & -0.03 & -0.71 & 0.474 & -0.13 & 0.06 \\
\hline
\end{tabular}

stimuli. More importantly, applying a whole-brain searchlight approach to target specificity of neural representations on the item level, we found clear evidence for age differences in itemspecific BOLD activation patterns in occipital regions.

Previous studies with young adults have established that reduced item specificity results in poor memory performance (Xue et al., 2010; Hasinski and Sederberg, 2016), but a predefined focus on category-specific regions in VVC (e.g., Koen et al., 2019) may have prevented previous age-comparative studies to reveal age differences in item-level specificity and delineate their role in cognitive decline. The current study demonstrates that age differences in item specificity are most prominent in occipital regions (including lateral occipital cortex and occipital face area for faces; Fig. 5), that are involved in early visual processing. Together with the fusiform gyrus, lateral occipital cortex has been shown to be critical for visual object (including face) perception (Grill-Spector et al., 1999; Grill-Spector and Malach, 2004; Nagy et al., 2012). Furthermore, the occipital face area, which is connected to the FFA and lateral occipital cortex, constitutes the lowest level in the face perception network and is suggested to represent and integrate facial components (Pitcher et al., 2007, 2011; Kadosh et al., 2011; Nagy et al., 2012). Thus, less precise item-specific activation patterns in older adults in these regions are likely to reflect differences in early processing of the incoming visual information, which results in detrimental consequences for downstream (category-level) processing. In sum, we argue that our results are in line with core assumptions of computational models (e.g., S. C. Li et al., 2001), namely, that increased neural variability with age manifests as both degraded item representations in early visual regions and, as a downstream effect, reduced category selectivity in VVC. Hence, we emphasize the important role of item-specific and stable neural representations for memory functioning.

Here, we only investigated cortical representations, but these may depend on pattern separation in the hippocampus, which is known to decline in aging (e.g., Reagh et al., 2018). Future research should study the interdependence of hippocampal and cortical representations and their joint association to memory performance during aging (compare Danker et al., 2017).

In contrast to previous studies (e.g., Koen et al., 2019; Srokova et al., 2020), we did not observe differences in agerelated neural dedifferentiation based on stimulus materials. However, the limited variety of different stimulus categories in our study does not allow to make strong claims for or against the proposition that neural dedifferentiation is ubiquitous in the aging brain. Differences between stimulus categories should thus be examined in a systematic investigation in the future, including a broad range of different stimulus categories and other factors, such as task demands.

\section{Interindividual and intraindividual differences in neural distinctiveness are related to memory performance}

Next, using LMM, we explored the unique effects of interindividual differences in three measures capturing neural dedifferentiation at distinct representational levels (category selectivity, category specificity, and item specificity) on memory performance. Importantly, of the neural dedifferentiation measures, item specificity showed the strongest association with memory, over and above the shared effects of the distinctiveness measures across all representational levels combined. Since the recognition memory task used in the current study required participants to distinguish between highly similar face and house exemplars, rather than simply discriminating faces from houses, high item specificity was required to perform well. Category selectivity instead may rather be a general indicator of representational quality and a potential downstream effect of item-specific perceptual representations. However, when tested by itself, category selectivity was also shown to be related to memory performance 
across participants. The finding that category selectivity was associated with recognition performance while category specificity was not was also observed by Srokova et al. (2020). However, in contrast to that, Koen et al. (2019) reported a link between recognition memory and both category selectivity and specificity. These deviant results may result from methodological differences, including stimulus materials (see also above) and task demands (incidental encoding vs intentional encoding). Overall, while our results nicely illustrate the coexistence of age differences at different representational levels, they clearly identify item specificity in early visual areas as the driving force for interindividual differences in memory performance.

Our results further revealed the importance of item specificity for the mnemonic fate of single items, that is, subsequent memory effects at the within-person level. In line with others, we defined item specificity as the comparison of activation patterns across stimulus repetitions in relation to their similarity to different stimuli of the same category (compare Hasinski and Sederberg, 2016; Zheng et al., 2018; Koen and Rugg, 2019). In that way, item specificity is a joint effect of representational stability and representational distinctiveness. Thus, theoretically, reduced item specificity in old age may be because of decreased item stability (Zheng et al., 2018), increased within-category similarity (Goh et al., 2010), or both (compare Koen and Rugg, 2019). However, the computational model by S. C. Li et al. (2001) suggests that item-level specificity in older adults is reduced because of increased neural response variability, thus reduced item stability. In line with this proposal, we observed that age differences in item specificity were because of a reduction in item stability in the older age group, whereas no age differences were observed for within-category similarity. Furthermore, item stability displayed an age-independent relation to memory, as indicated by a positive association with recognition probability, also on the trial level. Higher item stability in younger compared with older adults in visual regions being related to subsequent memory effects has also been reported by one previous age-comparative study (Zheng et al., 2018); however, the validity of that finding has been questioned (Koen and Rugg, 2019) because age differences in item specificity were not reliable. Our results clearly align with these prior results and highlight that item specificity and item stability are key contributors to age differences in memory performance.

The finding of impoverished item representations in older adults in early visual regions resonates with the long-standing suggestion in cognitive aging science that sensory and cognitive functions are increasingly linked to each other in the course of aging, which further reinforces the close connection of age-related deficits in perceptual processing and declining cognitive abilities (Lindenberger and Baltes, 1994; Baltes and Lindenberger, 1997; Schneider and Pichora-Fuller, 2000; K. Z. Li and Lindenberger, 2002). The current study substantiates this claim by identifying reduced representational specificity coexisting at different representational levels but most prominently in early visual regions, which was furthermore linked to interindividual and intraindividual differences in memory performance.

Although the current study clearly identifies a relationship between representational specificity and stability during encoding with later memory performance, the older adults group, who showed overall reduced specificity and stability, did not perform overall significantly worse than the young adult group, similar to findings by Koen et al. (2019). Less pronounced or no age-related deficits in item recognition memory, compared with, for example, associative memory, are not uncommon, especially under incidental encoding conditions (compare Old and Naveh-Benjamin, 2008;
Sommer et al., 2021). Nevertheless, older adults often tend to respond "old" more frequently than younger adults, contributing to their higher rates of false memories (Koutstaal and Schacter, 1997; Schacter et al., 1997; Fandakova et al., 2013, 2020), which was also observed in the present study. Nevertheless, the present study clearly shows that differences in representational specificity and stability during encoding can account for differences in subsequent memory performance between as well as within participants.

\section{Neural dedifferentiation as a consequence of age-related neurotransmitter changes?}

In the current study, we proposed that age differences at various representational levels coexist based on a computational model (S. C. Li et al., 2000, 2001; S. C. Li and Sikström, 2002) that suggests deficient neuromodulation as the causal mechanism for reduced consistency of neural information processing with ensuing consequences for representational distinctiveness and agerelated cognitive deficits. Of course, in the current study, the precise mechanism underlying the observed representational differences at the various levels remains speculative. Nevertheless, evidence from other studies supports the proposal of age differences in neuromodulation as a driving factor for neural dedifferentiation. While the original proposal emphasized age-related changes in dopamine (compare Wong et al., 1984; S. C. Li et al., 2001; Bäckman et al., 2006, 2010; Abdulrahman et al., 2017; Rieckmann et al., 2018), more recently, several studies have revealed the contribution of age-related decline in GABA to cognition and neural information representation (compare Leventhal et al., 2003). For example, using MR spectroscopy to quantify GABA concentrations in occipital voxels, Simmonite et al. (2019) found that lower occipital GABA levels in older adults were associated with declines in fluid processing abilities. Furthermore, Chamberlain et al. (2021) reported that individual differences in the category specificity of face versus house representations were linked to individual differences in VVC GABA concentrations in older adults, indicating a role of GABA in age-related neural dedifferentiation (see also Cassady et al., 2019; Gagnon et al., 2019; Lalwani et al., 2019). These findings substantiate the key role of proficient (particularly GABAergic) neuromodulation for high-fidelity (i.e., stable and distinct) neural representations and cognitive performance and lay the ground for future studies aiming to understand how agerelated deficits in neuromodulation are related to neural dedifferentiation across representational levels.

\section{Conclusion}

In conclusion, the present fMRI study set out to investigate age differences in neural information representation and their relation to episodic memory performance. Previous studies have focused on neural dedifferentiation of categorical information but have neglected age differences at the item level. Our results show that differences in representational distinctiveness coexist at various levels and contribute to memory. Crucially, they emphasize the striking relevance of item specificity and item stability for the understanding of interindividual and intraindividual differences in memory. Thus, the current study substantiates the claim of a close connection between age differences across processing levels (e.g., sensory and cognitive), as derived from long-standing observations in behavioral aging research. Longitudinal studies are needed to elucidate to what extent the different levels depend on or precede each other and whether a general mechanism (e.g., changes in neuromodulation) accounts for changes in representational properties. 


\section{References}

Abdulrahman H, Fletcher PC, Bullmore E, Morcom AM (2017) Dopamine and memory dedifferentiation in aging. Neuroimage 153:211-220.

Allen M, Poggiali D, Whitaker K, Marshall TR, Kievit RA (2019) Raincloud plots: a multi-platform tool for robust data visualization. Wellcome Open Res 4:63.

Bäckman L, Lindenberger U, Li SC, Nyberg L (2010) Linking cognitive aging to alterations in dopamine neurotransmitter functioning: recent data and future avenues. Neurosci Biobehav Rev 34:670-677.

Bäckman L, Nyberg L, Lindenberger U, Li SC, Farde L (2006) The correlative triad among aging, dopamine, and cognition: current status and future prospects. Neurosci Biobehav Rev 30:791-807.

Baltes PB, Lindenberger U (1997) Emergence of a powerful connection between sensory and cognitive functions across the adult life span: a new window to the study of cognitive aging? Psychol Aging 12:12-21.

Carp J, Park J, Polk TA, Park DC (2011) Age differences in neural distinctiveness revealed by multi-voxel pattern analysis. Neuroimage 56:736-743.

Cassady K, Gagnon H, Lalwani P, Simmonite M, Foerster B, Park D, Peltier SJ, Petrou M, Taylor SF, Weissman DH, Seidler RD, Polk TA (2019) Sensorimotor network segregation declines with age and is linked to GABA and to sensorimotor performance. Neuroimage 186:234-244.

Chamberlain JD, Gagnon H, Lalwani P, Cassady KE, Simmonite M Seidler RD, Taylor SF, Weissman DH, Park DC, Polk TA (2021) GABA levels in ventral visual cortex decline with age and are associated with neural distinctiveness. Neurobiology of Aging. Advance online publication. Retrieved February 15, 2021. doi: 10.1016/j.neurobiolaging.2021.02.013.

Corwin J (1994) On measuring discrimination and response bias: unequal numbers of targets and distractors and two classes of distractors. Neuropsychology 8:110-117.

Danker JF, Tompary A, Davachi L (2017) Trial-by-trial hippocampal encoding activation predicts the fidelity of cortical reinstatement during subsequent retrieval. Cerebral Cortex 27:3515-3527.

D’Esposito M, Zarahn E, Aguirre GK, Rypma B (1999) The effect of normal aging on the coupling of neural activity to the bold hemodynamic response. Neuroimage 10:6-14.

Dimsdale-Zucker HR, Ranganath C (2018) Representational similarity analyses: A practical guide for functional MRI applications. In: Handbook of Behavioral Neuroscience (Manahan-Vaughan D, ed), pp 509-525. Handbook of Neural Plasticity Techniques. San Diego: Elsevier.

Ebner NC, Riediger M, Lindenberger U (2010) FACES-a database of facial expressions in young, middle-aged, and older women and men: development and validation. Behav Res Methods 42:351-362.

Edelman S (1998) Representation is representation of similarities. Behav Brain Sci 21:449-467.

Epstein R, Kanwisher N (1998) A cortical representation of the local visual environment. Nature 392:598-601.

Esteban O, Markiewicz CJ, Blair RW, Moodie CA, Isik AI, Erramuzpe A, Kent JD, Goncalves M, DuPre E, Snyder M, Oya H, Ghosh SS, Wright J, Durnez J, Poldrack RA, Gorgolewski KJ (2019) fMRIPrep: a robust preprocessing pipeline for functional MRI. Nat Methods 16:111-116.

Fandakova Y, Shing YL, Lindenberger U (2013) High-confidence memory errors in old age: the roles of monitoring and binding processes. Memory 21:732-750.

Fandakova Y, Werkle-Bergner M, Sander MC (2020) (Only) time can tell: age differences in false memory are magnified at longer delays. Psychol Aging 35:473-483.

Folstein MF, Folstein SE, McHugh PR (1975) Mini-mental state: a practical method for grading the cognitive state of patients for the clinician. J Psychiatr Res 12:189-198.

Gagnon H, Simmonite M, Cassady K, Chamberlain J, Freiburger E, Lalwani P, Kelley S, Foerster B, Park DC, Petrou M, Seidler RD, Taylor SF, Weissman DH, Polk TA (2019) Michigan Neural Distinctiveness (MiND) study protocol: investigating the scope, causes, and consequences of age-related neural dedifferentiation. BMC Neurol 19:61.

Gazzaley AH, D'Esposito M (2005) BOLD functional MRI and cognitive aging. In: Cognitive neuroscience of aging: linking cognitive and cerebral aging (Cabeza R, Nyberg L, Park DC, eds), pp 107-131. Oxford: Oxford University Press.

Goh JO, Suzuki A, Park DC (2010) Reduced neural selectivity increases fMRI adaptation with age during face discrimination. NeuroImage 51:336-344.

Gorgolewski KJ, Auer T, Calhoun VD, Craddock RC, Das S, Duff EP, Flandin G, Ghosh SS, Glatard T, Halchenko YO, Handwerker DA,
Hanke M, Keator D, Li X, Michael Z, Maumet C, Nichols BN, Nichols TE, Pellman J, Poline JB, et al. (2016) The brain imaging data structure, a format for organizing and describing outputs of neuroimaging experiments. Sci Data 3:160044.

Grill-Spector K, Kushnir T, Edelman S, Avidan G, Itzchak Y, Malach R (1999) Differential processing of objects under various viewing conditions in the human lateral occipital complex. Neuron 24:187-203.

Grill-Spector K, Malach R (2004) The human visual cortex. Annu Rev Neurosci 27:649-677.

Grinband J, Steffener J, Razlighi QR, Stern Y (2017) BOLD neurovascular coupling does not change significantly with normal aging. Hum Brain Mapp 38:3538-3551.

Hasinski AE, Sederberg PB (2016) Trial-level information for individual faces in the fusiform face area depends on subsequent memory. Neuroimage 124:526-535.

Haynes JD, Rees G (2006) Decoding mental states from brain activity in humans. Nat Rev Neurosci 7:523-534.

Kadosh KC, Walsh V, Kadosh RC (2011) Investigating face-property specific processing in the right OFA. Soc Cogn Affect Neurosci 6:58-65.

Kanwisher N, McDermott J, Chun MM (1997) The fusiform face area: a module in human extrastriate cortex specialized for face perception. J Neurosci 17:4302-4311

Koen JD, Rugg MD (2019) Neural dedifferentiation in the aging brain. Trends Cogn Sci 23:547-559.

Koen JD, Hauck N, Rugg MD (2019) The relationship between age, neural differentiation, and memory performance. J Neurosci 39:149-162.

Koen JD, Srokova S, Rugg MD (2020) Age-related neural dedifferentiation and cognition. Curr Opin Behav Sci 32:7-14.

Koutstaal W, Schacter DL (1997) Gist-based false recognition of pictures in older and younger adults. J Mem Lang 37:555-583.

Kriegeskorte N, Formisano E, Sorger B, Goebel R (2007) Individual faces elicit distinct response patterns in human anterior temporal cortex. Proc Natl Acad Sci USA 104:20600-20605.

Kriegeskorte N, Mur M, Bandettini PA (2008) Representational similarity analysis: connecting the branches of systems neuroscience. Front Syst Neurosci 2:4.

Kuhl BA, Chun MM (2014) Successful remembering elicits event-specific activity patterns in lateral parietal cortex. J Neurosci 34:8051-8060.

Lalwani P, Gagnon H, Cassady K, Simmonite M, Peltier S, Seidler RD, Taylor SF, Weissman DH, Polk TA (2019) Neural distinctiveness declines with age in auditory cortex and is associated with auditory GABA levels. Neuroimage 201:116033.

Leventhal AG, Wang Y, Pu M, Zhou Y, Ma Y (2003) GABA and its agonists improved visual cortical function in senescent monkeys. Science 300:812-815.

Li KZ, Lindenberger U (2002) Relations between aging sensory/sensorimotor and cognitive functions. Neurosci Biobehav Rev 26:777-783.

Li SC, Sikström S (2002) Integrative neurocomputational perspectives on cognitive aging, neuromodulation, and representation. Neurosci Biobehav Rev 26:795-808.

Li SC, Lindenberger U, Frensch PA (2000) Unifying cognitive aging: from neuromodulation to representation to cognition. Neurocomputing 32:879-890.

Li SC, Lindenberger U, Sikström S (2001) Aging cognition: from neuromodulation to representation. Trends Cogn Sci 5:479-486.

Lindenberger U, Baltes PB (1994) Sensory functioning and intelligence in old age: a strong connection. Psychol Aging 9:339-355.

Lu H, Liu P (2017) MRI measures of aging: methodological issues. In: Cognitive neuroscience of aging: linking cognitive and cerebral aging, Ed 2 (Cabeza R, Nyberg L, Park DC, eds), pp 9-34. New York: Oxford University Press.

Mumford JA, Turner BO, Ashby FG, Poldrack RA (2012) Deconvolving BOLD activation in event-related designs for multivoxel pattern classification analyses. Neuroimage 59:2636-2643.

Mur M, Bandettini PA, Kriegeskorte N (2009) Revealing representational content with pattern-information fMRI: an introductory guide. Soc Cogn Affect Neurosci 4:101-109.

Nagy K, Greenlee MW, Kovács G (2012) The lateral occipital cortex in the face perception network: an effective connectivity study. Front Psychol 3:141. 


\section{This article has been retracted}

Nili H, Wingfield C, Walther A, Su L, Marslen-Wilson W, Kriegeskorte N (2014) A toolbox for representational similarity analysis. PLoS Comput Biol 10:e1003553.

Oostenveld P, Fries P, Maris E, Schoffelen JM (2011) FieldTrip: open source software for advanced analysis of MEG, EEG, and invasive electrophysiological data. Comput Intell Neurosci 2011:156869.

Old SR, Naveh-Benjamin M (2008) Differential effects of age on item and associative measures of memory: a meta-analysis. Psychol Aging 23:104118.

Paller KA, Wagner AD (2002) Observing the transformation of experience into memory. Trends Cogn Sci 6:93-102.

Park DC, Polk TA, Park R, Minear M, Savage A, Smith MR (2004) Aging reduces neural specialization in ventral visual cortex. Proc Natl Acad Sci USA 101:13091-13095.

Park J, Carp J, Hebrank A, Park DC, Polk TA (2010) Neural specificity predicts fluid processing ability in older adults. J Neurosci 30:9253-9259.

Park J, Carp J, Kennedy KM, Rodrigue KM, Bischof GN, Huang CM, Rieck JR, Polk TA, Park DC (2012) Neural broadening or neural attenuation? Investigating age-related dedifferentiation in the face network in a large lifespan sample. J Neurosci 32:2154-2158.

Payer D, Marshuetz C, Sutton B, Hebrank A, Welsh RC, Park DC (2006) Decreased neural specialization in old adults on a working memory task. Neuroreport 17:487-491.

Pitcher D, Walsh V, Duchaine B (2011) The role of the occipital face area in the cortical face perception network. Exp Brain Res 209:481-493.

Pitcher D, Walsh V, Yovel G, Duchaine B (2007) TMS evidence for the involvement of the right occipital face area in early face processing. Curr Biol 17:1568-1573.

Reagh ZM, Noche JA, Tustison NJ, Delisle D, Murray EA, Yassa MA (2018) Functional imbalance of anterolateral entorhinal cortex and hippocampal dentate/CA3 underlies age-related object pattern separation deficits. Neuron 97:1187-1198.e4.

Rieckmann A, Johnson KA, Sperling RA, Buckner RL, Hedden T (2018) Dedifferentiation of caudate functional connectivity and striatal dopamine transporter density predict memory change in normal aging. Proc Natl Acad Sci USA 115:10160-10165.

Rugg MD, Morcom MA (2005) The Relationship Between Brain Activity, Cognitive Performance, and Aging: The Case of Memory. In: Cognitive Neuroscience of Aging: Linking Cognitive and Cerebral Aging, pp 132154. New York, NY, US: Oxford University Press.

Schacter DL, Koutstaal W, Norman KA (1997) False memories and aging. Trends Cogn Sci 1:229-236.

Schneider BA, Pichora-Fuller MK (2000) Implications of perceptual deterioration for cognitive aging research. In: The handbook of aging and cognition, Ed 2, pp 155-219. Mahwah, NJ: Lawrence Erlbaum.
Simmonite M, Carp J, Foerster BR, Ossher L, Petrou M, Weissman DH, Polk TA (2019) Age-related declines in occipital GABA are associated with reduced fluid processing ability. Acad Radiol 26:1053-1061.

Snodgrass JG, Corwin J (1988) Pragmatics of measuring recognition memory: applications to dementia and amnesia. J Exp Psychol Gen 117:34-50.

Sommer VR, Mount L, Weigelt S, Werkle-Bergner M, Sander MC (2021) Memory specificity is linked to repetition effects in event-related potentials across the lifespan. Developmental Cognitive Neuroscience 48: 100926.

Sommer VR, Fandakova Y, Grandy TH, Shing YL, Werkle-Bergner M, Sander MC (2019) Neural pattern similarity differentially relates to memory performance in younger and older adults. J Neurosci 39:8089-8099.

Srokova S, Hill PF, Koen JD, King DR, Rugg MD (2020) Neural differentiation is moderated by age in scene- but not face-selective cortical regions. ENeuro 7:ENEURO.0142-20.2020.

St-Laurent M, Abdi H, Bondad A, Buchsbaum BR (2014) Memory reactivation in healthy aging: evidence of stimulus-specific dedifferentiation. J Neurosci 34:4175-4186.

Trelle AN, Henson RN, Simons JS (2019) Neural evidence for age-related differences in representational quality and strategic retrieval processes. Neurobiol Aging 84:50-60.

Voss MW, Erickson KI, Chaddock L, Prakash RS, Colcombe SJ, Morris KS, Doerksen S, Hu L, McAuley E, Kramer AF (2008) Dedifferentiation in the visual cortex: an fMRI investigation of individual differences in older adults. Brain Res 1244:121-131.

Ward EJ, Chun MM, Kuhl BA (2013) Repetition suppression and multi-voxel pattern similarity differentially track implicit and explicit visual memory. The Journal of Neuroscience 33:14749-14757.

West KL, Zuppichini MD, Turner MP, Sivakolundu DK, Zhao Y, Abdelkarim D, Spence JS, Rypma B (2019) BOLD hemodynamic response function changes significantly with healthy aging. Neuroimage 188:198-207.

Wilkinson GN, Rogers CE (1973) Symbolic description of factorial models for analysis of variance. J R Stat Soc C 22:392-399.

Wong DF, Wagner HN, Dannals RF, Links JM, Frost JJ, Ravert HT, Wilson AA, Rosenbaum AE, Gjedde A, Douglass KH (1984) Effects of age on dopamine and serotonin receptors measured by positron tomography in the living human brain. Science 226:1393-1396

Xue G (2018) The neural representations underlying human episodic memory. Trends Cogn Sci 22:544-561.

Xue G, Dong Q, Chen C, Lu Z, Mumford JA, Poldrack RA (2010) Greater neural pattern similarity across repetitions is associated with better memory. Science 330:97-101.

Zheng L, Gao Z, Xiao X, Ye Z, Chen C, Xue G (2018) Reduced fidelity of neural representation underlies episodic memory decline in normal aging. Cereb Cortex 28:2283-2296. 